\title{
Production of oestrone and oestradiol-17 $\beta$ by different regions of the filamentous pig blastocyst
}

\author{
L. A. Bate* and G. J. King \\ Department of Animal and Poultry Science, University of Guelph, Guelph, Ontario, \\ Canada N1G $2 \mathrm{Wl}$
}

\begin{abstract}
Summary. Pig blastocysts aged 14, 16 and 18 days were divided into $15 \mathrm{~cm}$ segments representing tissue adjacent to the embryonic disc, an intermediate section and the tip region. Whenever total blastocyst length allowed, the intermediate segment was divided into proximal and distal portions for separate culture. All were rinsed with buffer and incubated with dehydroepiandrosterone for $3 \mathrm{~h}$. Rinsing buffer and incubation medium were subsequently assayed for concentrations of oestrone and oestradiol-17 3 . The highest production of oestrogen was found in the embryonic disc region. The intermediate regions had the lowest synthetic ability, while the tip region produced more oestrogens than the intermediate regions but less than the disc region. The production of oestrone was higher $(P<0.05)$ in 18 -day-old blastocysts than in younger ones while oestradiol-17 $\beta$ production was lower $(P<0.05)$ on Day 16 . The proportional role of the embryonic disc region as oestrogen-producing tissue increased over time. On Day 14, each intermediate region produced over $70 \%$ as much oestrogen as the disc region. These proportions declined on Days 16 and 18 to about 50 and $30 \%$ respectively. The regional variation in the ability of blastocysts to produce oestrogens may have some influence on the ability of the blastocyst to create an adequate microenvironment within the uterus which permits successful differentiation and placentation.
\end{abstract}

Keywords: oestrogens; blastocyst; pigs; pregnancy; uterine environment

\section{Introduction}

There is abundant information suggesting that oestrogens play a significant role in signalling the endocrine transition from a cycling to pregnancy status in pigs (Bazer $\&$ Thatcher, 1977). The exact mechanism, however, is still unclear. The ability of the pig blastocyst to produce oestrogens was identified by Perry et al. (1973) and starts 10 or 12 days post coitum (Heap et al., 1975; Stone \& Seamark, 1985; King \& Ackerley, 1985). In addition to its antiluteolytic role, oestrogens have been functionally related to embryo migration within the uterus (Stone \& Seamark, 1985; Pope et al., 1986), to increased blood flow during the 2 nd and 3rd week of gestation (Ford, 1985), to embryo development (Dalton \& Knight, 1983) and to stimulation of the uterus. These events apparently influence the formation of a suitable microenvironment for differentiation and development of the blastocyst (Geisert et al., 1982; Morgan et al., 1987).

King \& Ackerley (1985) demonstrated regional differences in oestrone and oestradiol-17 $\beta$ amounts in blastocyst tissue between Days 10 and 16. Larger amounts were found in the yolk sac

*Present address: Department of Anatomy and Physiology, Faculty of Veterinary Medicine, University of Prince Edward Island, Charlottetown, Canada C1A 4P3. 
endoderm compared to trophectoderm during later stages. These regional differences may be crucial for stimulating the uterus at the proper time and in the correct sequence to promote successfully the adequate microenvironment conducive to blastocyst development. The lack of understanding about the role which different structures or regions within the pig blastocyst play in the biosynthesis of oestrogens is not surprising considering that most information about in-vitro production of oestrogens has been based on results from homogenized samples of the entire blastocyst or randomly selected tissue segments. The present study intended to investigate whether there were differences in the abilities of various regions of the pig blastocyst to synthesize oestrone and oestradiol-17 $\beta$ between Days 14 and 18 of gestation.

\section{Materials and Methods}

Pig blastocysts were collected from 24 crossbred females at $14(\mathrm{~N}=2), 16(\mathrm{~N}=19)$ or $18(\mathrm{~N}=3)$ days of gestation (day oestrus signs first observed = Day 0 ). The sows were stunned by electrocution, exsanguinated and the uteri immediately excised and moved to the laboratory in an insulated container. Within 10 min after the start of the slaughtering process, the mesometrium was detached and the uterine horns opened longitudinally along the antimesometrial border. The open horns were then transferred to a dissection tray, submerged in phosphate-buffered saline (pH 7.2, 0.1 M) (PBS) and pinned to the wax base with the mucosal side up. Separation of the blastocyst from the endometrial mucosa was carried out by stretching the endometrial folds and rinsing the chorionic vesicles loose with a gentle stream of buffer. The buffer was continuously recycled by filtering through a Radiometer circulating heater bath which was maintained at temperatures between 33 and $37^{\circ} \mathrm{C}$. After detachment, the filamentous blastocysts were extended to full length, measured and divided into regions, excluding the embryonic disc and the small but visible allantoic membrane attached to the Day-18 embryos. The trophoblast could not be separated from yolk sac tissue within any particular region but the yolk sac stalks and some of the adjacent membranes were eliminated when the Day-18 embryonic discs were detached. Segments $\sim 15 \mathrm{~cm}$ long were taken near the disc and tips. Whenever blastocysts were of sufficient length, the remaining intermediate tissue was divided to obtain middle region samples. In this manner duplicate sets of comparable segments (i.e. one collected from each side of the disc) were obtained from most blastocysts. Only two segments per side, designated as disc and tip regions, were obtained from blastocysts measuring up to $60 \mathrm{~cm}$. The $60-90 \mathrm{~cm}$ specimens were divided into disc, middle and tip regions. When the total length was $>90 \mathrm{~cm}$ the middle region could be further divided into a proximal segment designated as the middle-disc and the distal portion designated as the middle-tip region.

Excess fluid was allowed to drain from each segment, tissue pieces were weighed, minced, rinsed in $2 \mathrm{ml}$ PBS by gentle agitation, placed in a tube and maintained in a waterbath at $37^{\circ} \mathrm{C}$ until 2 or 3 embryos from the same animal were processed. All tubes were then centrifuged for $2 \min \left(500 \mathrm{~g}, 35^{\circ} \mathrm{C}\right)$ to separate tissue from the supernatant rinsing buffer which was then decanted and frozen for subsequent analysis. The tissue was resuspended in $4 \mathrm{ml}$ Eagle's minimal essential medium (GIBCO) and transferred to $5 \mathrm{ml}$ incubation flasks, with 20 ng dehydroepiandrosterone (DHA) added as an oestrogen precursor (Perry et al., 1973). The prepared flasks were incubated for $3 \mathrm{~h}$ at $37^{\circ} \mathrm{C}$ in an humidified environment gassed with $95 \%$ air and $5 \% \mathrm{CO}_{2}$ (Trujano \& Wrathall, 1985). Segments collected from a random sample of blastocysts were incubated at $4 . \mathrm{C}$ without DHA to serve as a basal control for the incubation procedure. After incubation, culture medium and tissue were separated and frozen in different vials. The concentration of oestrone and oestradiol- $17 \beta$ in rinsing buffers and incubation media and the protein content of the tissues were measured. Only those embryos which could be processed within 40 min from exsanguination to incubation were used, permitting a maximum of 3 blastocysts per animal.

A modification of the RIA described by Bate \& Hacker (1982) was used for oestrone measurement. The present assay used the same antibody but omitted the column purification step required for serum and the extraction solvent used was diethyl ether. The inter- and intra-assay coefficients of variation were 11.5 and $2.2 \%$ respectively. Assay of various dilutions of medium or rinsing buffer resulted in a parallel displacement of label from the antibody with respect to the standard curve.

Oestradiol-17 was quantified with an assay described by Ferket \& Hacker (1985) using an antiserum supplied by $\operatorname{Dr}$ H. A. Robertson. The cross-reactions with other steroids were: oestrone $4 \cdot 5 \%$, oestriol $0 \cdot 5 \%$, oestrone sulphate $0.2 \%$, oestradiol- $17 \alpha \quad 0.05 \%$ and testosterone, androsterone, dehydroepiandrosterone, androstenediol, epiandrosterone, progesterone, pregnenolone, cortisol, 11-desoxycortisol and corticosterone all at $<0.01 \%$. The inter- and intra-assay coefficients of variation for oestradiol-17ß were $12 \cdot 1$ and $9 \cdot 2 \%$ respectively. Assay of various dilutions of medium or rinsing buffer resulted in a parallel displacement of label from the antibody with respect to the standard curve. The oestradiol- $17 \beta$ assay for the rinsing samples did not include the solvent extraction procedure used in culture samples as the correlation coefficient between measured concentrations of oestradiol-17 $\beta$ in 24 extracted and non-extracted samples was 0.96 . The percentage of oestradiol-17 $\beta$ detected without extraction was $97 \%$ of that measured after solvent extraction. Four atypical blastocysts were excluded from analysis (those with hormone measurements of more than twice the standard deviation of the mean value), including a blastocyst measuring $2300 \mathrm{~mm}$, which was the only blastocyst in the uterus of a sow with a total of 4 corpora lutea. 
Tissue samples were homogenized in $2 \mathrm{ml}$ PBS and total protein was measured using a commercial protein assay kit (Bio-Rad Laboratories, Mississauga). The coefficient of variation for this assay, determined by 12 replicates within the run, was $5 \cdot 12 \%$. When more than two sections were obtained from a middle portion the values were pooled into middle-disc and middle-tip regions to standardize all blastocysts to a maximum of 4 regions. Although there was a significant correlation $(r=0.75)$ between the concentration of oestrogens expressed in $\mathrm{pg} / \mathrm{mg}$ wet tissue and $\mathrm{pg} / \mathrm{mg}$ protein, the values are expressed in relation to protein to eliminate some of the random variation generated by different quantities of fluid retained by blastocysts during collection and transfer to culture tubes.

The statistical analysis was performed with SAS using general linear models (Spector et al., 1985). Comparison of oestrogen production by different regions was performed independently for each age group and separately for each embryo length. Categories in which only one blastocyst was represented were excluded from this independent analysis. The model used in the analysis included the hormonal concentration in rinsing buffer or incubation medium as the dependent variable and the position of the section, the sow and the embryo nested within sows as the independent variables. Oestrogen production between age groups was compared using total embryonic production. Comparison between oestrone and oestradiol- $17 \beta$ values as well as between culture media and their respective rinsing component was done by $t$ test. Duncan's multiple-comparison range test was used for comparison of individual regional means.

\section{Results}

The embryonic disc region had the highest synthesizing ability for both oestrone and oestradiol-17 $\beta$ $(P<0.05)$ in embryos of all the ages studied. Oestrogen production decreased $(P<0.05)$ in the intermediate regions and was again elevated in the tip region (Fig. 1), which nevertheless remained below the values of the embryonic disc region $(P<0.05)$. A significant difference $(P<0.001)$ between the very low concentration of oestrogens produced by tissue incubated in medium only $(294 \pm 24$ and $30 \pm 8 \mathrm{pg} / \mathrm{mg}$ protein for oestrone and oestradiol-17 $\beta$, respectively) and the markedly elevated amounts when the medium was fortified with DHA precursor $(760 \pm 47$ and $85 \pm 6 \mathrm{pg} / \mathrm{mg}$ protein for oestrone and oestradiol-17 $\beta$, respectively) confirmed that the culture system was effective in sustaining the in-vitro oestrogen synthetic abilities of the tissues. There was a large sow effect $(P<0.001)$ in the oestrogen content and production by blastocyst within any of the days studied.

Comparison of the oestrogens found in the rinsing buffer of each piece of blastocyst before incubation and the quantity synthesized during incubation and recovered in the culture medium revealed only a moderate correlation $(r=0 \cdot 34 ; P<0 \cdot 05)$. Both oestrone and oestradiol-17 $\beta$ were relatively evenly distributed throughout all regions of the blastocyst before incubation (Fig. 1).

As time progressed, the overall oestrogen synthetic ability of the embryonic disc region became proportionally higher than that in the other regions. For oestrone, this trend was mainly due to a net increase in the production rate of the disc region on Days 16 and 18 , concurrently with a modest decrease in the rate of oestrone produced by the intermediate regions. For oestradiol-17 $\beta$, the proportionally higher synthesis by the disc region was the result of a net decrease in the production by the middle and tip regions (Fig. 1). This distribution throughout the blastocyst was maintained despite a generalized decrease in oestradiol-17 $\beta$ synthesis by all regions of the Day-16 blastocysts.

Mean oestrone production on Day $18(907 \pm 163 \mathrm{pg} / \mathrm{mg}$ protein) was higher $(P<0.05)$ than on Days 14 and $16(729 \pm 52$ and $687 \pm 47 \mathrm{pg} / \mathrm{mg}$ protein, respectively) but oestradiol-17 $\beta$ was lower $(P<0.05)$ on Day $16(66 \pm \mathrm{pg} / \mathrm{mg}$ protein $)$ when compared to Days 14 and $18(154 \pm 16$ and $83 \pm 16 \mathrm{pg} / \mathrm{mg}$ protein, respectively). The lower production of the middle region compared with the disc and tip regions is better illustrated by a separate analysis applied only to the groups of blastocysts measuring about $30,45,60 \mathrm{~cm}$ or more, from the disc to one tip within each age group (Table 1). This allowed comparison of duplicate segments collected from each side of the disc yielding only disc and tip regions if total length was $<60 \mathrm{~cm}$; disc middle and tip regions in blastocysts between 60 and $90 \mathrm{~cm}$; and disc, middle-disc, middle-tip and tip regions from larger blastocysts. It is possible that shorter blastocysts have the same distribution of oestrogens as longer blastocysts, but the number of pieces collected from shorter blastocysts did not permit distinction of separate middle regions. 

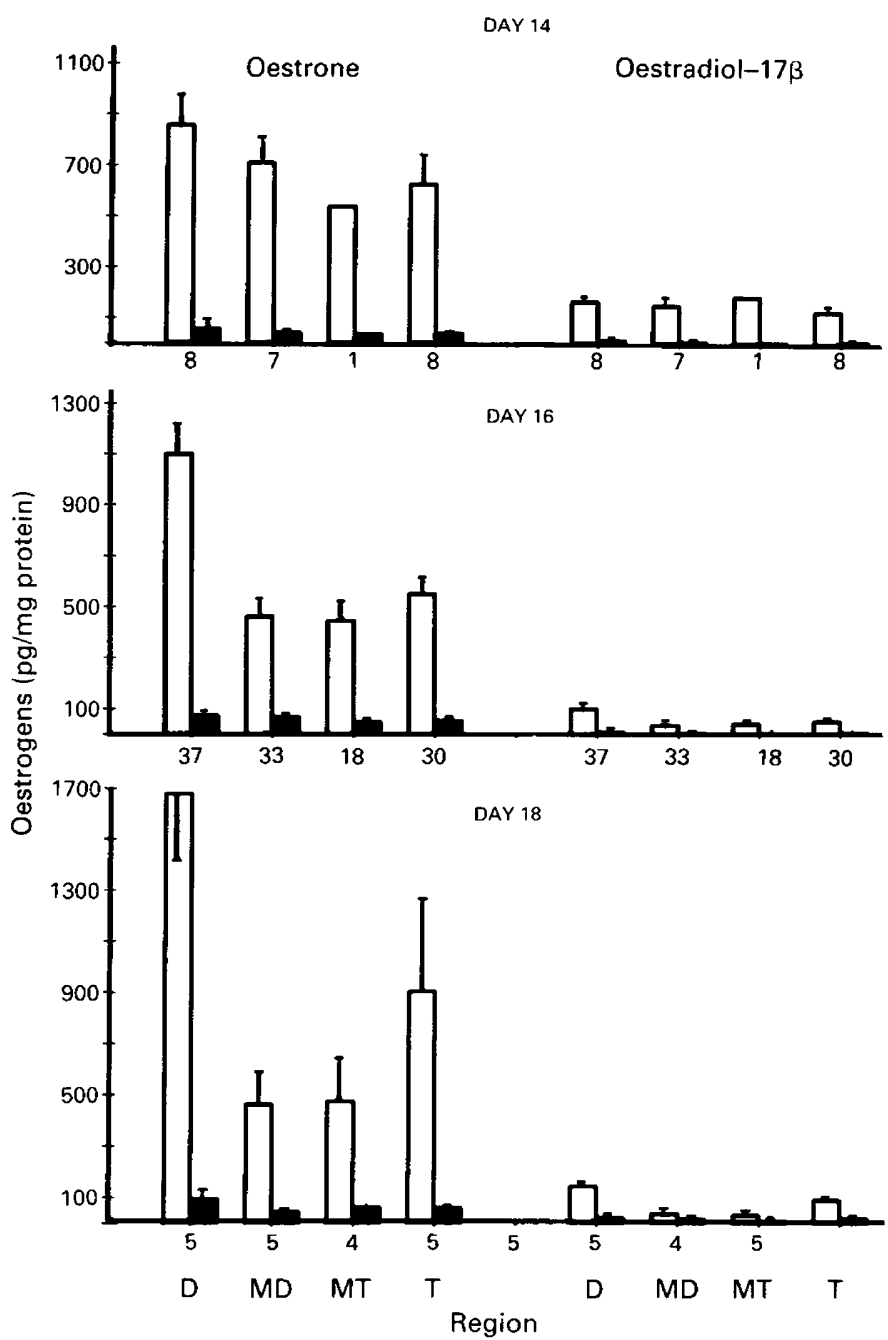

Fig. 1. Concentrations (mean \pm s.d.) of oestrone and oestradiol-17 $\beta$ in different regions of $14-$ 18-day-old pig blastocysts. Solid bars represent oestrogens present in tissue rinsings before incubation. Open bars represent concentrations and hence production of oestrogens after a 3-h incubation with dehydroepiandrosterone as precursor. $\mathrm{D}=15-\mathrm{cm}$ region proximal to disc; $\mathrm{MD}$ and $\mathrm{MT}=$ middle sections closer to the disc and tip respectively; $\mathrm{T}=15-\mathrm{cm}$ region including the trophoblast tip. The no. of samples represented is indicated.

\section{Discussion}

Previous studies on oestrogen production by pig conceptuses (Perry et al., 1973; Heap et al., 1975; Guthrie \& Lewis, 1986) have dealt with all the embryonic membranes as a single unit. The structural differences existing in composition of the chorionic vesicle membrane in various regions between the embryonic disc and the tip prompted an investigation of oestrogen synthesis by the various segments. The mesoderm is present in pig conceptuses beneath and beside the disc but has not spread to $5 \mathrm{~cm}$ away by Day 14 . Subsequent mesodermal proliferation results in yolk sac 


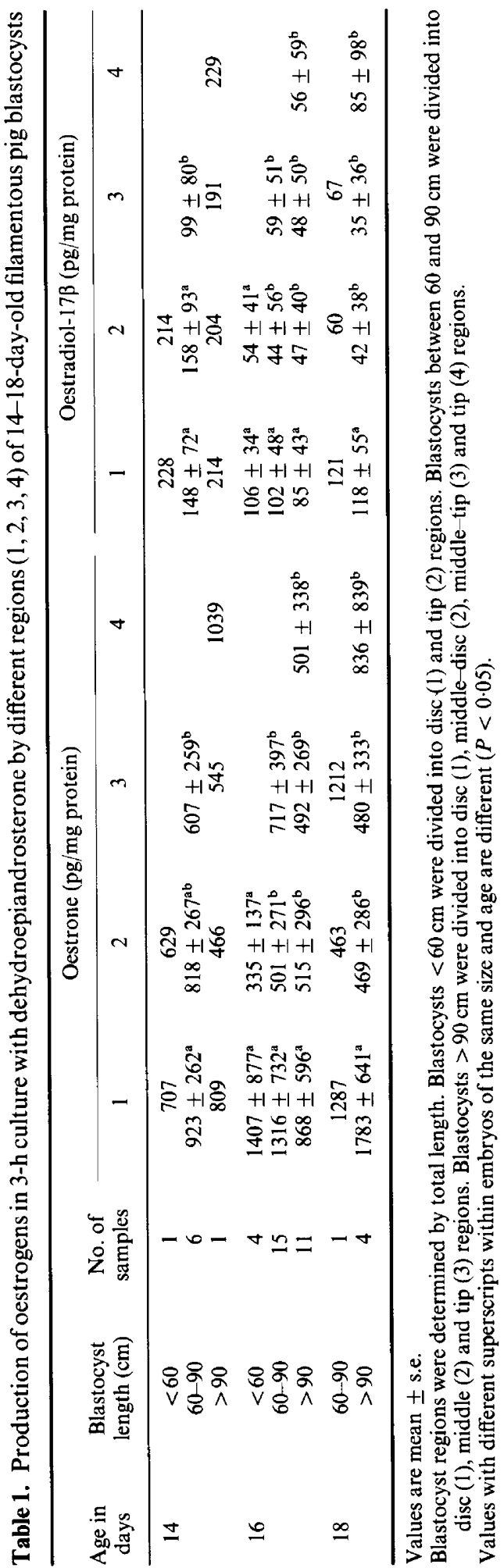


formation which may extend to $20 \mathrm{~cm}$ or more from the disc by Days 16 and 18 . Oestrone and oestradiol- $17 \beta$ could be localized in both the trophectoderm and the yolk sac endoderm during the 2nd and 3rd week of development but the intensity of the immunocytochemical reaction indicated that the later membrane may be the major site of oestrogen synthesis during Days 14-18 (King \& Ackerly, 1985). The present study demonstrated that the different regions of the blastocyst have unequal abilities for oestrogen synthesis and the presence of the yolk sac endoderm in the disc region would account for the greater production. Since placentation begins at the embryonic disc and spreads rapidly towards the chorionic tip (King et al., 1982), these changes in steroidogenic capacity of different regions within individual chorionic vesicles may be important in the local regulation leading to successful placentation and survival.

Elaborate precautions were taken to avoid chilling and to get material into culture as quickly as possible. This was done with the expectation that it would preserve cellular viability and promote maximum steroidogenesis. However, the concentrations produced during the culture period were not higher than those reported by Perry et al. (1976) or Guthrie \& Lewis (1986), who chilled tissues before culture and so precautions taken to prevent temperature shock were not essential. However, culturing of individual regional segments has substantially reduced the variability of oestrogen measured, probably due to the similarity in the make up of the tissue forming each region studied.

The relatively low correlation between oestrogens measured in the original rinsing buffer and culture medium after incubation may be partly explained by free diffusion of oestrogens throughout the central cavities of the tubular blastocyst. Therefore, although the disc area is producing more steroids during the stages studied, they apparently diffuse freely and accumulate throughout the blastocyst in a relatively even manner. The great difference in oestrogens produced by different sows could be a consequence of a difference of $\pm 24 \mathrm{~h}$ in chronological age between litters or possibly a genetic paternal influence which has been found in pigs (L. A. Bate \& R. R. Hacker, unpublished data). The increase in oestrone by Day 18 and the fluctuation of oestradiol-17 $\beta$ between Days 14 and 18 coincides with earlier findings (Gadsby \& Heap, 1978). An optimum uterine microenvironment is required for embryonic development (Geisert et al., 1982; Morgan et al., 1987) and the differential rate of oestrogen production by individual regions of the blastocyst may provide a local stimulus for adjacent endometrium. The possibility that differential endocrine support may be required within a single blastocyst is reasonable since each blastocyst covers about $10 \mathrm{~cm}$ of unstretched uterus with about $100 \mathrm{~cm}$ of highly convoluted trophectodermal tube (Perry et al., 1976). This extensive regional coverage could facilitate secretory or physical differentiation of local microenvironments. Under this scheme, failure of some regions to supply oestrogens in adequate amounts and at the proper time may result in deficient developmental conditions which could lead to early embryonic mortality.

We thank V. Jasper-Fayer for technical assistance; and the Natural Sciences and Engineering Research Council of Canada and the Ontario Ministry of Agriculture and Food for financial support.

\section{References}

Bate, L.A. \& Hacker, R.R. (1982) Estrogen and piglet viability. I. Serum estrogen concentrations in piglets. J. Anim. Sci. 54, 1012-1016.

Bazer, F.W. \& Thatcher, W.W. (1977) Theory of maternal recognition of pregnancy in swine based on oestrogen controlled endocrine versus exocrine secretion of prostaglandin $\mathrm{F}_{2} \alpha$ by the uterine endometrium. Prostaglandins 14, 397-401.

Dalton, D.L. \& Knight, J.W. (1983) Effects of exogenous progesterone and estrone on conceptus development in swine. J. Anim. Sci. 56, 1354-1361.
Ferket, S.L. \& Hacker, R.R. (1985) Effect of forced exercise during gestation on reproductive performance of sows. Can. J. Anim. Sci. 65, 851-859.

Ford, S.P. (1985) Maternal recognition of pregnancy in the ewe, cow and sow: vascular and immunological aspects. Theriogenology 23, 145-159.

Gadsby, J.E. \& Heap, R.B. (1978) Steroid hormones and their synthesis in the early embryo. In Novel Aspects of Reproductive Physiology, pp. 263-285. Eds G. H. Spilman \& J. W. Wilks. J. Wiley \& Sons, Toronto.

Geisert, R.D., Renegar, R.H., Thatcher, W.W., Roberts, 
R.M. \& Bazer, F.W. (1982) Establishment of pregnancy in the pig: I. Interrelationships between preimplantation development of the pig blastocyst and uterine endometrial secretions. Biol. Reprod. 27, 925-939.

Guthrie, H.D. \& Lewis, G.S. (1986) Production of prostaglandin $F_{2} \alpha$ and estrogen by embryonal membranes and endometrium and metabolism of prostaglandin $\mathrm{F}_{2} \alpha$ by embryonal membranes, endometrium and lung from gilts. Dom. Anim. Endocr. 3, 185-198.

Heap, R.B., Perry, J.S., Gadsby, J.E. \& Burton, R.D. (1975) Endocrine activities of the blastocyst and the early embryonic tissue in the pig. Biochem. Soc. Trans. 3, 1183-1188.

King, G.J. \& Ackerley, C.A. (1985) Demonstration of oestrogens in the developing pig trophectoderm and yolk sac endoderm between Days 10 and $16 . J$. Reprod. Fert. 73, 361-367.

King, G.J., Atkinson, B.A. \& Robertson, H.A. (1982) Implantation and early placentation in domestic ungulates. J. Reprod. Fert., Suppl. 31, 17-30.

Morgan, G.L., Geisert, R.D., Zavy, M.T., Shawley, R.V. \& Fazleabas, A.T. (1987) Development of pig blastocysts in a uterine environment advanced by exogenous oestrogen. J. Reprod. Fert. 80, 125-131.
Perry, J.S., Heap, R.B. \& Amoroso, E.C. (1973) Steroid hormone production by pig blastocysts. Nature, Lond. 245, 45-47.

Perry, J.S., Heap, R.B., Burton, R.D. \& Gadsby, J.E. (1976) Endocrinology of the blastocyst and its role in the establishment of pregnancy. J. Reprod. Fert., Suppl. 25, 85-104.

Pope, W.F., Lawyer, M.S. \& First, N.L. (1986) Intrauterine migration of the porcine embryo: coordination of bead migration with estradiol. J. Anim. Sci. 63, $848-853$.

Spector, P., Goodnight, J.H., Sall, J.P. \& Sarle, W.S. (1985) The GLM procedure. In SAS/State Guide for Personal Computers Version 6 Edition. SAS Institute Inc. Cary, NC.

Stone, B.A. \& Seamark, R.F. (1985) Steroid hormones in uterine washings and in plasma of gilts between Days 9 and 15 after oestrus and between Days 9 and 15 after coitus. J. Reprod. Fert. 75, 209-221.

Trujano, M. \& Wrathall, A.E. (1985) Observations on the development in vitro of early (13-day) and later (15day) porcine embryos. Br. vet. J. 141, 378-387.

Received 24 December 1987 